\title{
MiR-143 suppresses the epithelial-mesenchymal transition of spinal glioblastoma through down-regulation of ERK5
}

\author{
Yi Yan ${ }^{1}$, Jing $\mathbf{W u}^{2}$, Mingjun $\mathbf{W u}^{3}$, Yongzhi Xia ${ }^{1}$, Wenyuan Tang ${ }^{1}$, Zhengbu Liao ${ }^{1}$ \\ ${ }^{1}$ Department of Neurosurgery, The First Affiliated Hospital of Chongqing Medical University, Chongqing, 400016, China \\ ${ }^{2}$ Department of Intensive Care Unit, The Second Affiliated Hospital of Chongqing Medical University, Chongqing, 400010, China \\ ${ }^{3}$ Institute of Molecular Biology, Chongqing Medical University, Chongqing, 400016, China \\ Correspondence to: Zhengbu Liao, email: liaozhengbu163@163.com
}

Keywords: miR-143, ERK5, spinal GBM

Received: September 14, 2016

Accepted: October 12, 2016

Published: October 28, 2016

\section{ABSTRACT}

The spinal glioblastoma (GBM) represents the one of most common tumors in humans. However, the biological processes and molecular mechanisms of spinal GBM are still unclear. It is known that miR-143 participates in the development of various tumor progressions. The present study was to evaluate the level of miR-143 in spinal GBM tissues and cells. We further investigated that the molecular mechanisms of miR143 in U87 and U251 cell lines. Here, our data showed that the expression levels of miR-143 were significantly reduced in spinal GBM tissues and cell lines. Accordingly, the expression levels of the extracellular signal regulated kinase 5 (ERK5) were significantly increased in spinal GBM tissues and cell lines. Ectopic expression of miR-143 in U87 and U251 cells resulted in decreased cell growth and enhanced cell apoptosis, and inhibited the expression of epithelial-mesenchymal transition (EMT) biomarkers. Further study characterized the 3' untranslated region (3'-UTR) of ERK5 gene as a direct target of $\mathrm{miR}-143$ in U87 and U251 cells as determined by luciferase reporter assays. In addition, the ectopic expression of miR-143 led to the downregulation of epidermal growth factor receptor (EGFR), while the over-expression of ERK5 reversed the miR-143-inhibited EGFR expression, and promoted cell growth and the expression of EMT biomarkers. In conclusion, this study demonstrated that miR-143 plays a crucial role in regulating the EMT of GBM by directly targeting ERK5, and miR-143 may act as a potential therapeutic target in spinal GBM patients.

\section{INTRODUCTION}

Intramedullary spinal cord tumors indicated a set of spinal tumors, which aroused from cells within the spinal cord. Spinal intramedullary tumors have been reported to account for about $15 \%$ in all spinal cord tumors, involving ependymomas, astrocytomas and hemangioblastomas [1, 2]. In general, spinal intramedullary tumors are featured as retarded progression and well differentiated property [3-6]. Recently, some study found that high-grade intramedullary tumors like anaplastic astrocytomas (Grade III) and glioblastomas (Grade IV) also occurred in the spinal cord. In recent decades, related mechanisms were little reported, and the treatment option is limited to the surgery. Thus, it is essential to find the relevant molecular etiology of spinal GBM.
MicroRNAs act as a kind of small and non-coding RNAs with the length of about 20 nucleotides, and take part in the regulation of gene expression. Mechanically, miRNAs can bind the 3'-unstranslated regions of the related target mRNA sequences, and then suppresses gene transcription or facilitate the degradation of mRNA [7]. To date, miRNAs have been demonstrated to play important roles in a wide range of oncogenic activities, including proliferation, apoptosis, angiogenesis, and metastasis [8]. Although the miRNA-induced potential regulation mechanisms are still under investigation, some current reports have indicated that the expression model of miRNA will be useful for the diagnosis and prognosis of human tumors [9]. Increasing studies identified that miR-143 can be found to be down-/up-regulated in diverse cancer tissues. At the same time, it has been reported that 
miR-143 has a wide role in regulation of some functions involving suppression of cancer cell proliferation and inhibition of organ metastasis of different cancers. As reported, the expression of miR-143 was found to be down-regulated in some cancers, inducing prostate cancer, colorectal neuroendocrine tumors, ovarian cancer and nonsmall cell lung cancer. However, little is known about the role of miR-143 in the development of GBM.

In the present study, our team demonstrated that the expression of miR-143 was down-regulated in spinal GBM tissues and GBM cell lines. In-vitro assays verified that over-expression of miR-143 in U87 and U251 cells led to a decrease in GBM cell proliferation and EMT biomarkers, and an increase in GBM cell apoptosis. Additionally, ERK5 was demonstrated as a direct target of miR-143. Furthermore, over-expression of ERK5 attenuated miR-143-inhibited cell biology. Overall, these findings suggested that miR-143 might be a potential oncogene in the development of spinal GBM.

\section{RESULTS}

\section{The expression of miR-143 in human spinal GBM samples and cell lines}

Firstly, we detected the expression status of miR-143 in all fifteen cases of spinal GBM samples and their paired adjacent non-tumor samples using RT-PCR analysis. Our findings revealed that the expression of miR-143 in tumor samples was significantly lower as compared with that in the their adjacent non-tumor samples $(\mathrm{p}<0.001)$ (Figure 1a). Statistically, the expression of miR-143 in most of samples in non-tumor samples displayed nearly 4-fold higher as compared with that in cancer samples, indicating that low expression of miR-143 might be involved in the development of human spinal GBM. Subsequently our team also detected the expression status of miR-143 in representative GBM U87 and U251 cell line using RTPCR analysis (Figure 1b). The results from U87 and U251 cells revealed the expression level of miR-143 was significantly lower as compared with that of human NHA cells.

\section{The expression of ERK5 is elevated in GBM samples and cell lines}

Based on the results above, our team further explored the expression level of ERK5 in human spinal GBM samples and cell lines using RT-PCR analysis and western blot analysis. Our data obtained from RTPCR analysis showed that compared with the non-tumor samples, the expression of ERK5 mRNA was significantly up-regulated in the GBM tumor samples. Likewise, high expression of ERK5 was also observed in U87 and U251

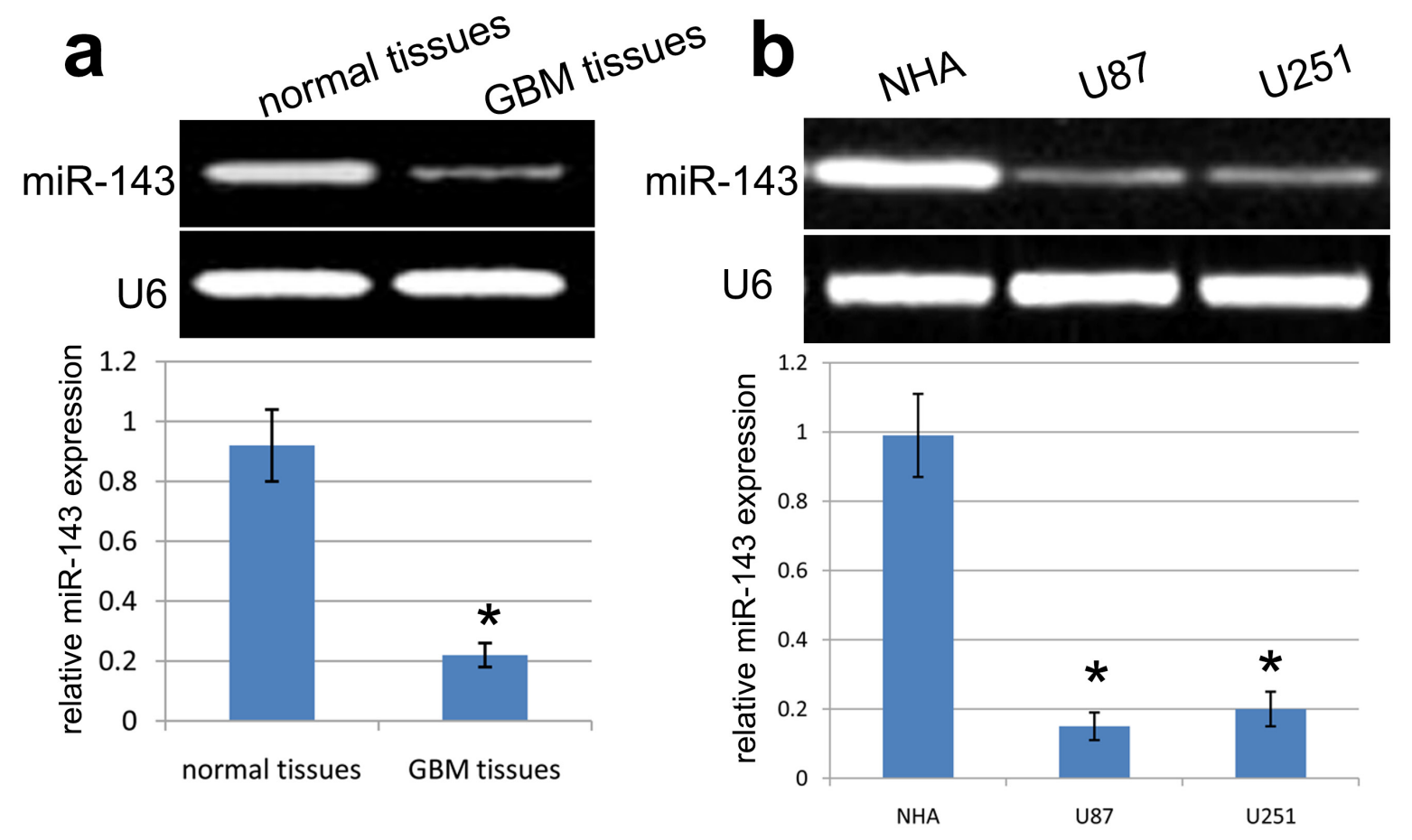

Figure 1: The expression profile of miR-143 in spinal GBM tissues and cell lines. a. MiR-143 expression was measured by RTPCR in normal tissues and spinal GBM tissues. All expression was normalized to the level of U6 small nuclear RNA expression. * $P<0.001$, compared with normal tissues, Students' t test. b. RT-PCR analysis indicated that the expression level of miR-143 was significantly lower in U87 and U251 cells compared to NHA cells. All expression was normalized to the level of U6 small nuclear RNA expression. ${ }^{*} P<0.001$, compared with NHA cells, one-way ANOVA. 
cell line as compared with human normal NHA cells (all $\mathrm{p}<0.001$ ) (Figure 2a, 2b). Data obtained from western blot analysis revealed that the expression of ERK5 protein was also significantly up-regulated in spinal GBM tumor tissues as well as U87 and U251 cell lines (all p<0.001) (Figure 3a, 3b). Combined with these analysis data, we calculated the correlation between miR-143 and ERK5, and observed that the expression of miR-143 was inversely proportional to that of ERK $5 \mathrm{mRNA}$ or protein $(\mathrm{r}=-0.811$, $p=0.002 ; r=-0.856, p=0.009$; respectively). Our findings indicated that miR-143 and ERK5 is involved into the development of human spinal GBM.

\section{MiR-143 mediates cell growth in GBM cells}

To investigate the biological functions and molecular mechanisms of miR-143 in GBM cells, we up-regulated the expression level of miR-143 by transiently transfecting

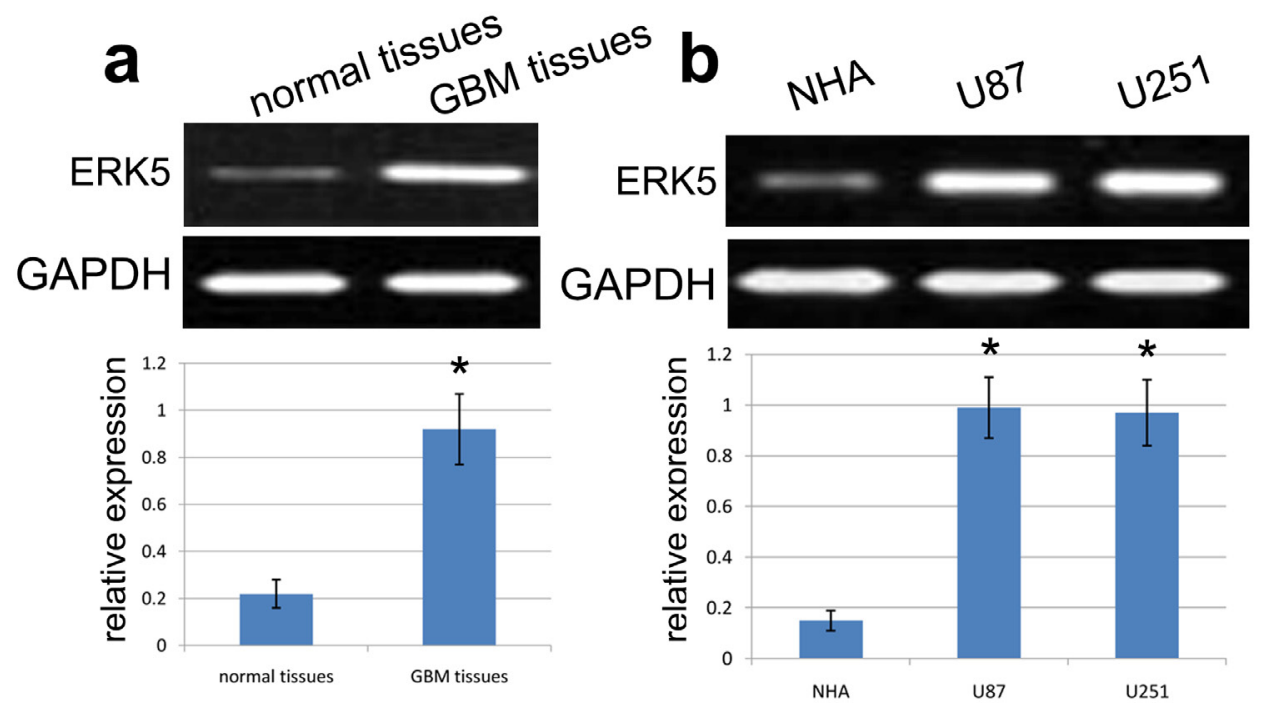

Figure 2: ERK5 is up-regulated in spinal GBM tissues and cell lines. a. RT-PCR analysis demonstrates that ERK5 expression was significantly increased in spinal GBM tissues compared with normal tissues. ${ }^{*} P<0.001$, compared with normal tissues, Students' $t$ test. b. RT-PCR analysis demonstrates that ERK5 expression was significantly increased in U87 and U251 cells compared with normal NHA cells. ${ }^{*} P<0.001$, compared with NHA cells, one-way ANOVA.

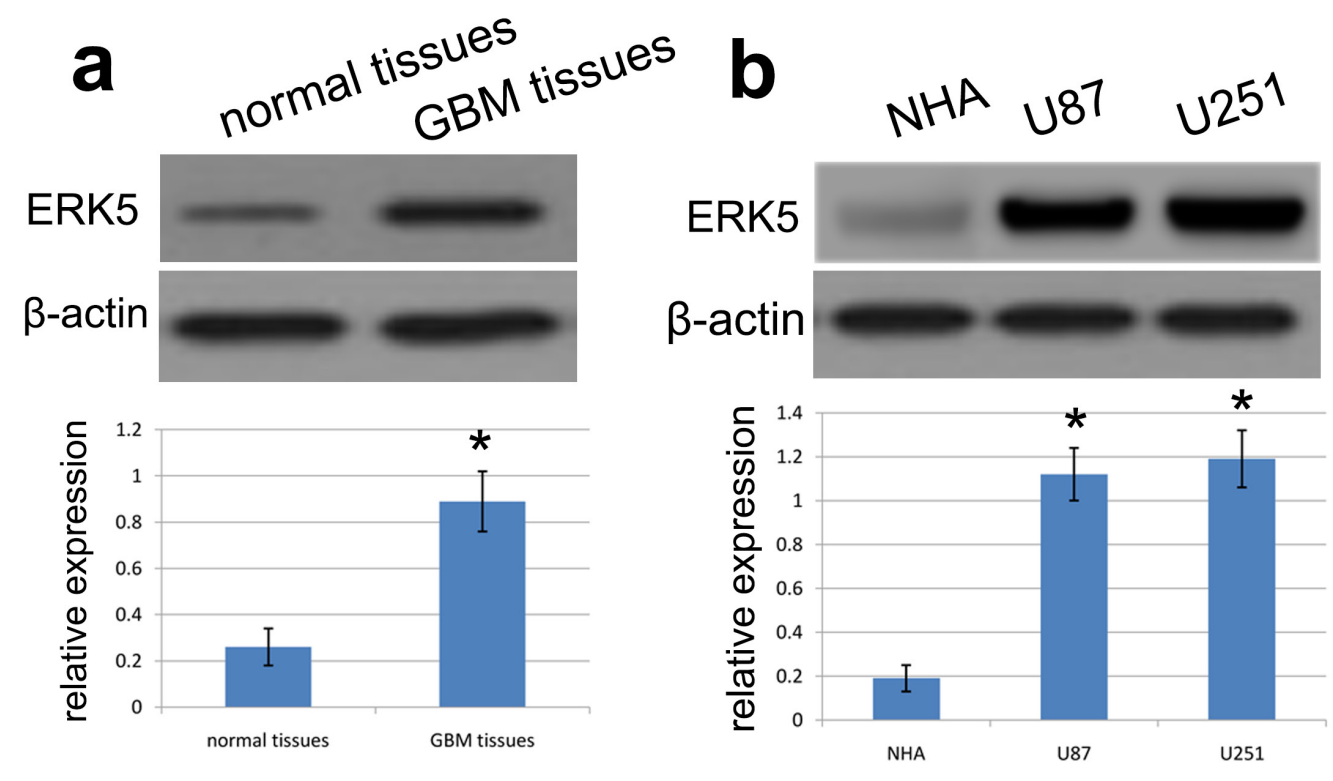

Figure 3: ERK5 is up-regulated in spinal GBM tissues and cell lines. a. Western blot analysis demonstrates that ERK5 expression was significantly increased in spinal GBM tissues compared with normal tissues. * $P<0.001$, compared with normal tissues, Students' $t$ test. b. Western blot analysis demonstrates that ERK5 expression was significantly increased in U87 and U251 cells compared with normal NHA cells. $* P<0.001$, compared with NHA cells, one-way ANOVA. 
cells with miR-143 mimics or NC miRNAs. As shown in Figure $4 \mathrm{a}$, as compared with NC miRNAs (miR-NC), U87 and U251 cells transfected with miR-143 mimics exhibited a significant decrease in the expression of miR143 using RT-PCR analysis. Following overexpression of miR-143, we used MTT assay to examine the impact of miR-143 on U87 and U251 cell growth, and demonstrated that over-expression of miR-143 obviously reduced U87 or U251 cell growth as compared to U87 or U251 cells with transfection of miR-NC (Figure 4b). These findings indicated that miR-143 was able to repress GBM cell growth.

\section{MiR-143 mediates cell apoptosis in GBM cells}

Next, we used flow cytometry to examine the impact of miR-143 on U87 and U251 cell apoptosis, and demonstrated that apoptosis was obviously altered in U87 or U251 cells with transfection of miR-143 mimics or miR-NC. The apoptotic rate of U87 and U251 cells transfected with miR-143 mimics was (11.3 \pm 2.2$) \%$ and $(15.4 \pm 3.1) \%$ respectively; On the other hand, The apoptotic rate of U87 and U251 cells transfected with miR-NC was $(3.3 \pm 1.0) \%$ and $(5.6 \pm 1.6) \%$, respectively (Figure 4c), which indicated that overexpression of miR143 facilitated cell apoptosis of U87 and U251 cells.

\section{MiR-143 mediates EMT in GBM cells}

In view of the importance of EMT in tumorigenesis, our team detected the expression status of the EMT biomarkers (MMP2, Slug, and N-cadherin) in U87 and U251 cells using western blot analysis. Data from western blot analysis showed that the expression level of MMP2, Slug and N-cadherin protein in U87 and U251 cells transfected with miR-143 mimics was significantly decreased, while the expression level of MMP2, Slug and $\mathrm{N}$-cadherin protein in U87 and U251 cells transfected with miR-NC was not altered (Figure 4d). This finding suggested that miR-143 inhibited EMT of GBM cells. Consistent with the results of MTT, we also found that the expression of EGFR was also affected by overexpression of miR-143 in U87 and U251 cells. In view of importance of EGFR in tumor growth, our results indicated that miR143 indeed suppressed cell growth in the development of GBM.

\section{ERK5 is a direct target of miR-143}

At first, we detected the expression of ERK5 using western blot analysis, and observed that the expression of ERK5 protein was greatly inhibited by the overexpression of miR-143 as compared with the control (Figure 4d). Afterwards, our team successfully constructed the 3'-UTR of ERK5 which contains predicted binding site of miR143. Using the luciferase reporter assay, we found that miR-143 significantly suppressed the luciferase activity of mutant 3'-UTR of ERK5 in U87 and U251 cells (Figure 5a), while miR-143 did not affected the luciferase activity of the wild type 3'-UTR of ERK5 in U87 and U251 cells. Taken together, these findings suggested that miR-143 directly targeted the expression of ERK 5 by binding its 3'UTR in GBM cells.

\section{Enforced ERK5 reverses miR-143-induced inhibition of EMT and growth}

To further investigate the relationship between miR-143 and ERK5, we enforced the expression of ERK5 by transfecting cells with ERK5 plasmids. To date, the importance of ERK5 has been reported widely in a wide range of human malignant cancer cells, we also explored the biological role of miR-143 in U87 and U251 cells. In this work, U87 and U251 cells co-transfected with full length ERK5 plasmids and miR-143 mimics exhibited a significant increase in the expression of ERK5 protein using Western blot analysis as compared with cells with vectors and miR-143 mimics (Figure 5b). Additionally, the expression of EMT biomarkers and EGFR was also affected in comparison with cells with vectors and miR143 mimics. And then, we investigated the role of ERK5 over-expression to the growth of U87 and U251 cell transfected with miR-143 mimics using MTT assay, and observed that enforced ERK5 expression significantly attenuated miR-143 mimics-inhibited cell growth of U87 and U251 cells as compared to cells with vector and miR143 mimics (Figure 6). These findings suggested that enforced ERK5 expression indeed reverses miR-143induced inhibition of EMT and growth.

\section{DISCUSSION}

miRNA was reported as one class of small and noncoding RNA, which has a very conservative sequence and structure. Functionally, miRNA is able to control the posttranscription expression of target genes by binding 3'-UTR of related genes [7]. A couple of studies have indicated that the expression of miR-143 was demonstrated to be down-regulated in a wide range of malignancies, such as cervical cancer, bone tumors and nasopharyngeal cancer [10-13]. Thus the tumor suppressor of miR-143 may be recommended as a new therapy method for spinal GBM patients. In this study, we assumed that miR-143 plays an oncogenic role in the development of GBM. According to the results, we found the expression of miR-143 was lowered in all tissues and samples of spinal GBM patients. At the same time, the expression of miR-143 was also down-regulated in GBM U87 and U251 cell lines. Because the generation of mature miRNA needs two basic processed, including the cleavage of endogenous transcript pri-miRNA by Drosha enzyme and the cleavage of the single stranded miRNA by Dicer enzyme, the deregulation of any factors like Drosha and Dicer enzyme will result 

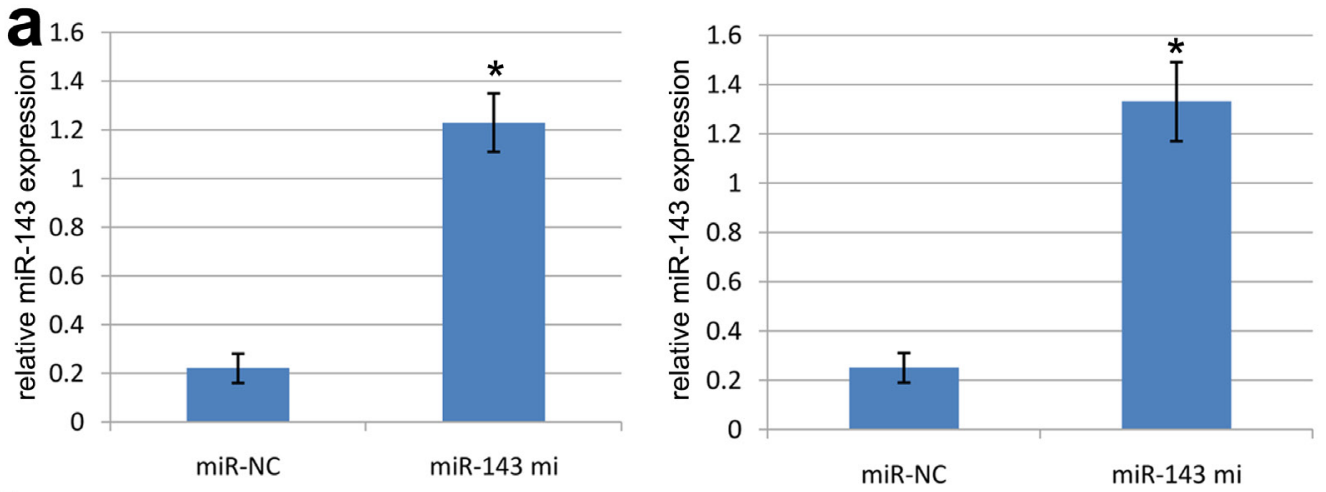

b
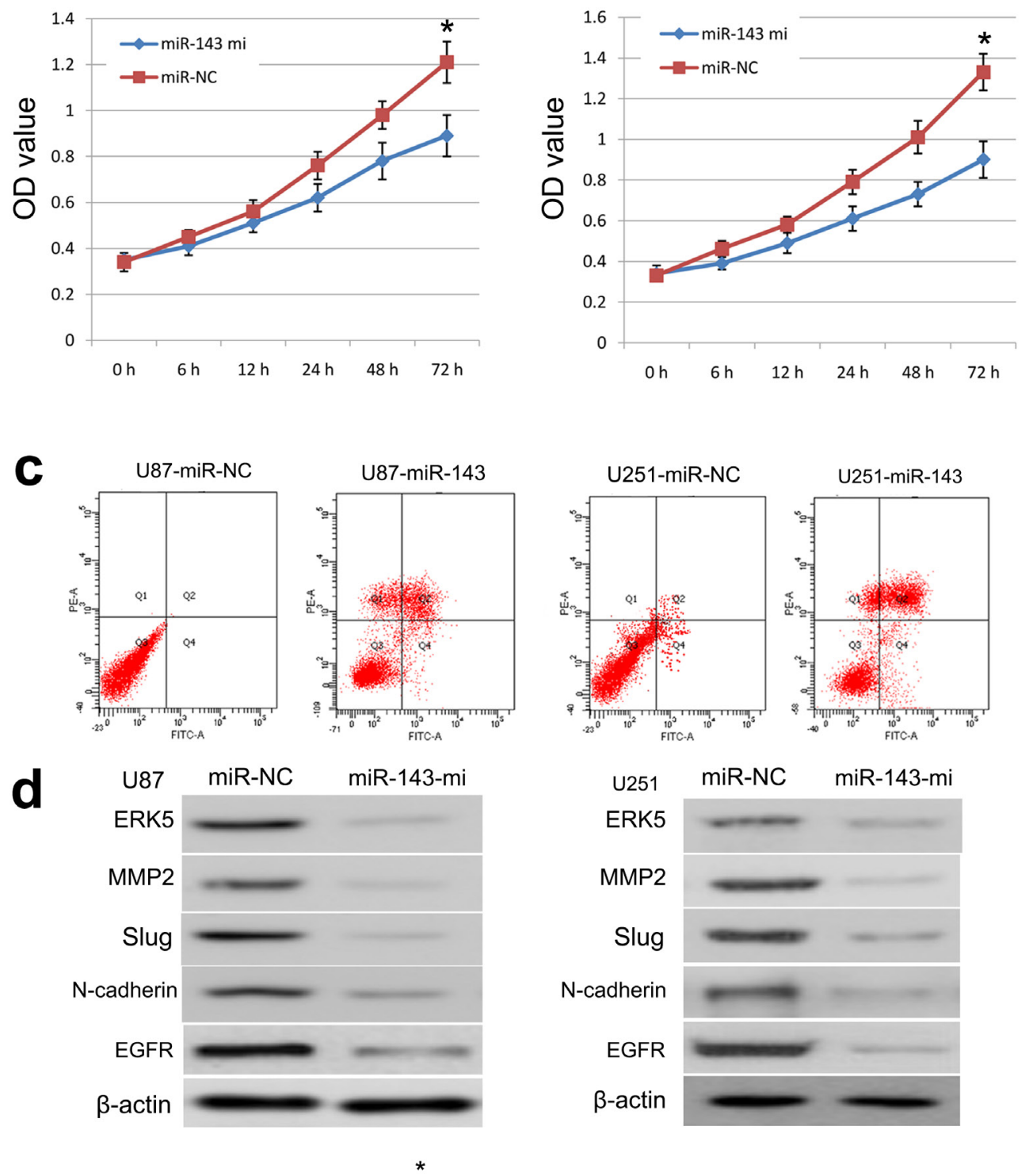

Figure 4: miR-143 over-expression promotes cell apoptosis and inhibited EMT. a. U87 and U251 cells were transfected with miR-143 mimics compared to U87 and U251 cells transfected with miR-NC. * $P<0.001$. b. Effect of miR-143 inhibition on U87 and U251 cell growth as measured by MTT assay. ${ }^{*} P<0.001$. c. The apoptosis of U87 and U251 cells was performed by flow cytometry after the transfection of miR-143 mimics or miR-NC. The graph shows the statistic data. The number of apoptotic cells was significantly higher in U87 and U251 cells over-expressing miR-143 as measured by PI staining and flow cytometry. d. Western-blots revealed that ERK5, EMT biomarkers and EGFR protein levels were markedly decreased in miR-143 mimics-transfected U87 and U251 cells compared to miR-NCtransfected cells. 


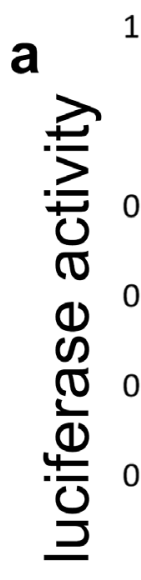

1.2

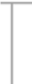

1

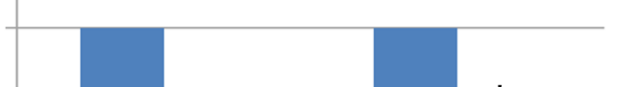

0.8

0.4

4

0.2

0

U87
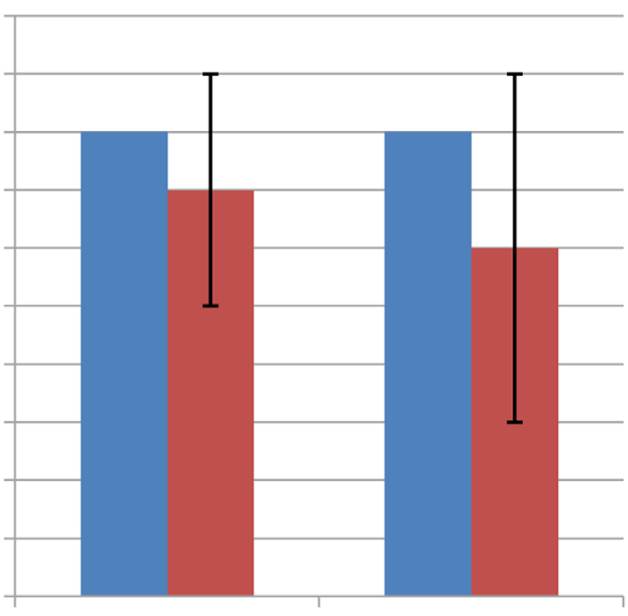

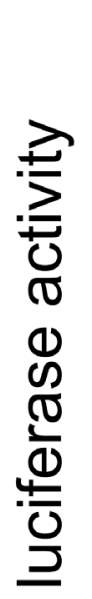

1.02

1.01

1

0.98

0.97

0.96

0.95

0.94

0.93

0.92

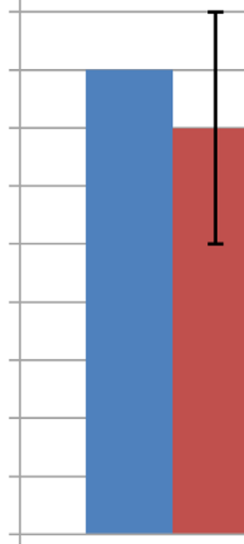

U87

b $\cup 87$

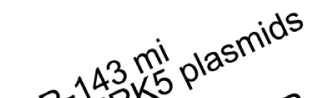

43 mi

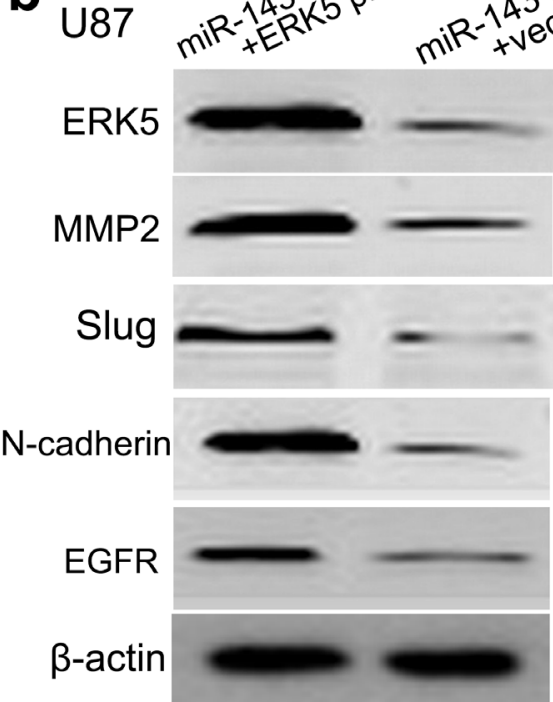

ERK5-3'UTR-mut

miR-143 mi

miR-NC

miR-143 mi

U251

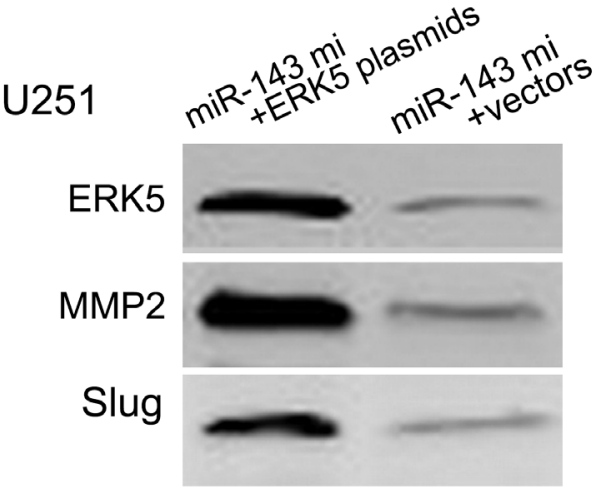

$\mathrm{N}$-cadherin

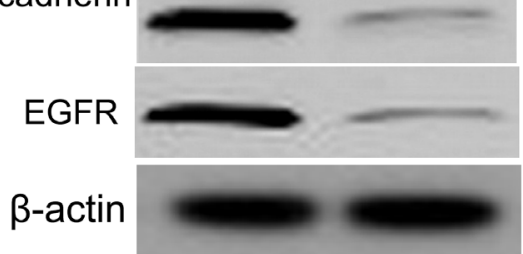

Figure 5: The miR-143 targets the 3'-UTR of ERK5 to regulate the EMT. a. A significant decrease in the relative luciferase activity was observed following co-transfection of ERK5-3'-UTR-wt with the miR-143 mimics, rather than ERK5-3'-UTR-mut with the miR-143 mimics. $* P<0.001$, compared with NC miRNAs, one-way ANOVA. b. Western blot analysis of ERK5, EMT biomarkers and EGFR protein levels after the co-transfection of miR-143 mimics with ERK5 plasmids or vectors to U87 and U251 cells. The relative expression level of proteins was measured by $\beta$-actin served as an internal control. 
in the down-regulation of miR-143. Overall findings obtained from this work showed that down-regulation of miR-143 were implicated into the development of GBM.

Functionally, miRNAs were demonstrated to broaden the epigenetic ranges in cells, and also altered the epigenetic models which were based on the level of chromatin previously [14-16]. As for miR-143, it regulated the expression of epigenetics-related genes, and took part in different cellular signalings $[17,18]$. In addition, miRNAs can bind the 3'-unstranslated regions (3'-UTR) of the related target mRNA sequences, and then suppresses gene transcription or facilitate the degradation of mRNA [7]. To date, miRNAs have been demonstrated to play important roles in a wide range of oncogenic activities, including proliferation, apoptosis, angiogenesis, and metastasis [8]. Although the miRNA-induced potential regulation mechanisms are still under investigation, some current reports have indicated that the expression model of miRNA will be useful for the diagnosis and prognosis of human tumors [9]. In the present study, our findings demonstrated that over-expression of miR-143 indeed enhanced GBM cell apoptosis. To figure out the molecular mechanisms of apoptosis mediated by miR143, we detected the expression of ERK5 gene and protein and EMT biomarkers as well as the expression of EGFR, and observed that over-expression of miR-143 affected the expression of these biomarkers in U87 and U251 cells, inferring that ERK5 and miR-143 have a potential relationship.

In view of results mentioned above, we selected the target gene ERK5, which plays an important role in the cell cycle and cell growth. ERK5 acts as one of members of the MAPK family, and has a conserved Thr-Glu-Tyr (TEY) structure in its phosphorylation sites. Consistent with the traditional ERK1/2, ERK5 plays a crucial role in the balance of cell growth and differentiation [19-21]. It is should be noted that ERK5 was also involved in the regulation of the proliferation and apoptosis in GBM cells. Here, we demonstrated that the regulation of miR143 in the expression of ERK5 in GBM cells. According to results, our team demonstrated that ERK5 was a direct down-stream target of miR-143, by which miR-143 is able to inhibit the expression of ERK5 and promote cell apoptosis in the development of GBM. Additionally, the inhibition of miR-143 would significantly increase the expression of ERK5 mRNA. Besides, we also demonstrated that overexpression of ERK5 could reverse miR-143 induced inhibition of GBM cell growth. In light of findings above, we believed that overexpression of miR-143 may be a useful and effective strategy for clinical treatment of spinal GBM patients by inhibition of ERK5 expression.

In conclusion, our findings showed that the expression of miR-143 were significantly down-regulated in spinal GBM samples and cells. In addition, we demonstrated that ERK5 is a novel target of miR-143. Mechanically, over-expression of miR-143 induced cell apoptosis through inhibition of ERK5-EMT pathway. Thus, miR-143 plays an important role in malignant progression of cancers, and miR-143 might represent a potential target for spinal GBM patients.
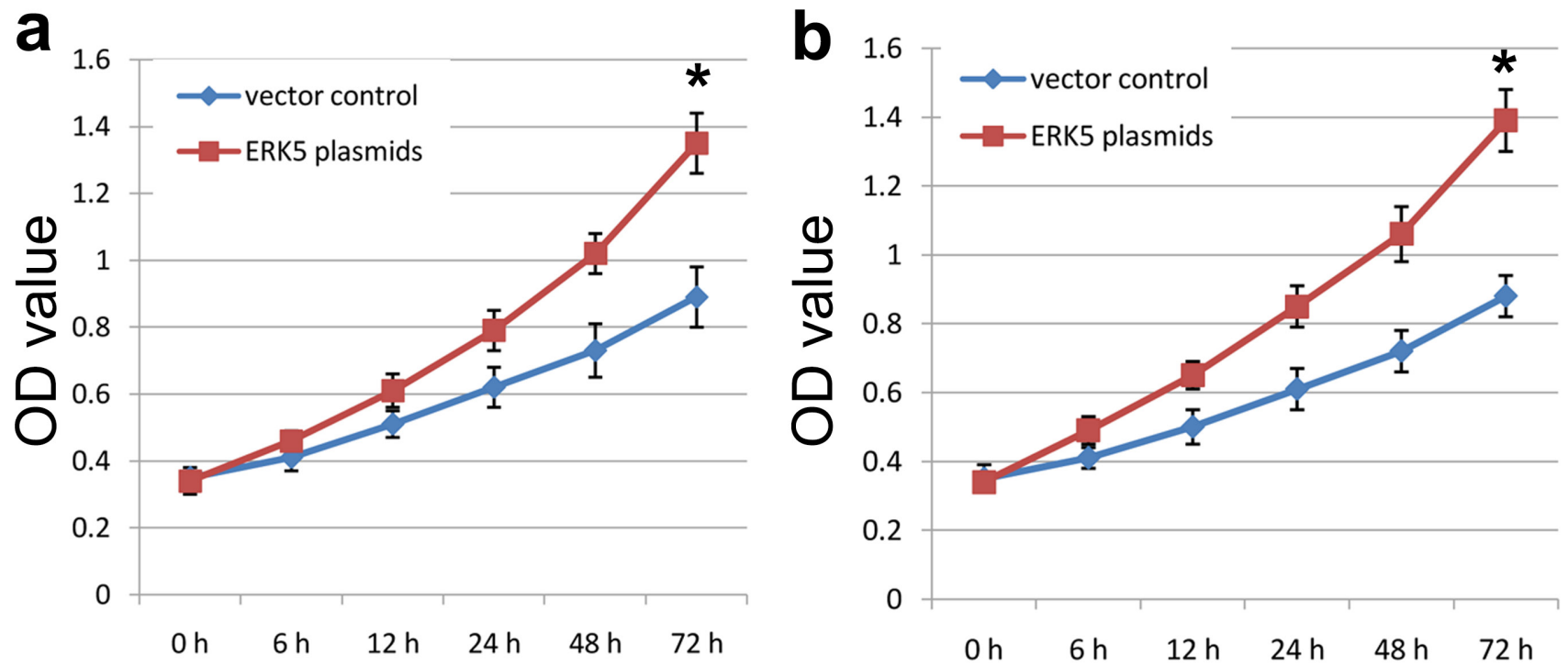

Figure 6: Over-expression of ERK5 reverses the inhibition of cell growth. Effect of ERK5 over-expression on the proliferation of U87 a. and U251 b. cells co-transfected with miR-143 mimics and ERK5 plasmids was measured by MTT assay. * $P<0.001$, compared with control, one-way ANOVA. 


\section{MATERIALS AND METHODS}

\section{Tissue samples}

Human tissue acquisition and use in this study complied with the National Regulations on the Use of Clinical Samples in China. 15 cases of tissue specimens were obtained from archived tissue samples from patients with spinal GBM who underwent surgical treatment at the First Affiliated Hospital of Chongqing Medical University from January 2008 to December 2015. A portion of the tumor tissues were saved and made into paraffin sections for histopathologic diagnosis in strict accordance with World Health Organization (WHO) criteria by two established neuropathologists, with differences resolved by careful discussion. And the remaining tissue was snapfrozen in liquid nitrogen then stored at $-80^{\circ} \mathrm{C}$ for RNA extraction and other biological molecular experiments. Before the RNA extraction from frozen tissues, the adjacent tumor tissues were subjected to frozen sections and reviewed by a pathologist to ensure that a minimum of $80 \%$ tumor cells were included in the sample. For spinal GBM patients, none of them had received chemotherapy or radiotherapy prior to surgery, and all patients were well followed up. Patients, who died of diseases not directly related to their gliomas or due to unexpected events, were excluded from this study. The present study was approved by the Ethics Committee of the First Affiliated Hospital of Chongqing Medical University.

\section{Cell culture}

As cell culture, primary normal human astrocytes (NHA) were purchased from the Sciencell Research Laboratories (Carlsbad, CA) and cultured under the conditions as instructed by the manufacturer. Cell lines U87 and U251 were obtained from the KeyGEN Company (China) and cultured in Dulbecco's Modified Eagle's medium (DMEM), 10\% fetal bovine serum (Gibco, Grand Island, NY, USA) and 1\% penicillin-streptomycin (Gibco, Grand Island, NY, USA) at $37^{\circ} \mathrm{C}$ in a humidified atmosphere under 5\% CO2. The medium was replaced every 3 days.

\section{Plasmids and miRNAs transfection}

Transfection was as described previously performed. Briefly, exponentially growing cells were seeded at $1 \times 10^{6}$ per $100 \mathrm{~mm}$ plate or at $1 \times 10^{5}$ per 96 -well plate overnight until $50 \%$ confluent. ERK5 plasmids, vectors, miR143 mimics, and non-targeting controls (miR-NC) were placed. Complete medium, together with the mixture from the above were then added to the plates after removing the old medium. ERK 5 constructs were purchased from Lifetechnologies (California, USA) and transfected using the manufacturer's reagents and protocol. Silencing of target gene expression was determined by Western blot analysis. Cells were incubated at $37^{\circ} \mathrm{C}$ with $5 \% \mathrm{CO} 2$ for $24 \mathrm{~h}$ with antibiotic-free medium.

\section{RT-PCR analysis}

Total RNAs were isolated from the cells by using Trizol reagent (TaKaRa, Japan). The sequences of primers used in this study were

MiR-143:

Forward: 5'-TGTAGTIGGAAGTGTCGCGC-3', Reverse: 5'-CCTACGATCGAAAACGACGCCAA CG-3';

ERK5:

Forward: 5'-CCTGAAGCCTA CTGTGCCCTATG-3', Reverse: 5'-CCGAAGCAGCT GGTACAGGAA-3'; U6:

Forward: 5'-CTCGCTTCGGCAGCACA-3', Reverse: 5'-AACGCTTCACGAATTTGCGT-3'; GAPDH:

Forward: 5'-CAATGACCCCTTCATTGACC-3', Reverse: 5'-GACAAGCTTCCCGTTCTCAG-3'; Total RNA $(1 \mu \mathrm{g})$ was reverse-transcribed into cDNA using a Quantscript RT Kit (Tiangen, China). PCR was performed using a PCR MasterMix Kit (BioTeke, China) in a GeneAmp PCR system 9600 (ABI Int.). cDNA was amplified under the thermocycling conditions as follows: 3 min initial denaturation at $94^{\circ} \mathrm{C}$ ( 1 cycle $)$, $30 \mathrm{~s}$ denaturation at $94^{\circ} \mathrm{C}$ ( 35 cycles), $30 \mathrm{~s}$ annealing at $57^{\circ} \mathrm{C}$, and $45 \mathrm{~s}$ extension at $72^{\circ} \mathrm{C}$. The last amplification was followed by final $7 \mathrm{~min}$ incubation at $72^{\circ} \mathrm{C}$. PCR products were separated by electrophoresis through $1 \%$ agarose gel, stained with ethidium bromide, and visualized by UV transillumination in a Tocan Gel Imaging System (Tocan Co., Shanghai, China). GAPDH or U6 was used as an internal control. The mRNA level was calculated by determining the integrated intensity of the bands of each treated group as a ratio of the control. Each sample was measured in triplicate.

\section{Western blot analysis}

For the protein analysis, the cells were harvested at 12 24 $\mathrm{h}$ following different treatments, as described above, and washed with cold PBS and then incubated in ice-cold RIPA buffer. Cell lysates were sonicated for $30 \mathrm{~s}$ on ice and lysed at $4^{\circ} \mathrm{C}$ for $60 \mathrm{~min}$. Then, the cell lysates were centrifuged at $12,000 \mathrm{~g}$ for $30 \mathrm{~min}$ at $4^{\circ} \mathrm{C}$. Protein concentrations in the supernatants were determined by the $\mathrm{BCA}$ reagent. Total protein was separated by denaturing $8-12 \%$ SDS-polyacrylamide gel electrophoresis, which was resolved over and electrotransferred by semidry blotting (Bio-Rad Laboratories, Shanghai) onto a nitrocellulose membrane. The membrane was incubated with antibodies ERK5, MMP2, Slug, N-cadherin, and EGFR (Abcam, Cambridge, UK, 1:1000 dilution) or 
$\beta$-actin (Santa Cruz Biotech, Santa Cruz, CA, 1:1000 dilution) overnight at $4{ }^{\circ} \mathrm{C}$, and then with peroxidaseconjugated secondary antibody (Santa Cruz Biotech, Santa Cruz, CA, 1:1000 dilution), visualized by chemiluminescence (GE, Fairfield, CT, USA).

\section{Cell proliferation assay}

Cell viability was assessed by the 3-(4, 5-dimethylthiazol-2-yl)-2, 5- diphenyltetrazolium bromide (MTT) assay. All cells were plated in 96-well plates at $4 \times 103$ cells/well and incubated with $0.2 \mathrm{mg} / \mathrm{ml} \mathrm{MTT} \mathrm{for}$ $4 \mathrm{~h}$ at $37^{\circ} \mathrm{C}$. The optical density of each sample was read using a microplate reader (BioRad) at $570 \mathrm{~nm}$.

\section{Apoptosis assay}

ApoScreen Annexin V Apoptosis Kit (Bender Med System, CA) were used for labeling of apoptotic cells. For each experiment, 20,000 cells were analyzed using FACSCalibur flow cytometer (BD Biosciences). Experiments were performed in triplicate.

\section{Luciferase reporter assay}

A dual-luciferase reporter vector was used to generate the luciferase constructs. For 3'-UTR luciferase assay, the putative binding sites of miR-143 and its homologous mutation sites in the 3'-UTR region of ERK5 mRNA were amplified and cloned into pGL3contral luciferase reporter plasmid (Invitrogen, Carlsbad, $\mathrm{CA})$. The pRL vector constitutively expressing Renilla luciferase was used to normalize for trasfection efficiency. Luciferase activity was measured using the DualLuciferase Reporter Assay System (Promega, Madison, USA) after transfection at $48 \mathrm{~h}$. Data are presented as the mean value \pm standard deviations (SD) for triplicate experiments.

\section{Statistics}

All data are presented as mean $\pm \mathrm{SD}$ of three separate experiments. Statistical analysis was performed using Student's $t$ test. $p<0.05$ was considered statistically significant. Analyses were performed with the SPSS 10.0 software (SPSS, USA).

\section{ACKNOWLEDGMENTS}

This work was supported by Chongqing Natural Science Foundation (cstc2015jcyjA0526).

\section{CONFLICTS OF INTEREST}

The authors declare no conflicts of interest.

\section{REFERENCES}

1. Bouffet E, Pierre-Kahn A, Marchal JC, Jouvet A, Kalifa C, Choux M, Dhellemmes P, Guérin J, Tremoulet M, Mottolese C. Prognostic factors in spinal cord astrocytoma. Cancer. 2000; 83:2391-2399.

2. Govindan A, Chakraborti S, Mahadevan A, Chickabasavaiah YT, Santosh V, Shankar SK. Histopathologic and immunohistochemical profile of spinal glioblastoma: a study of six cases. Brain Tumor Pathol. 2011; 28:297-303.

3. Henson JW. Spinal cord gliomas. Curr Opin Neurol 2001; 14:679-682.

4. Liu X, Germin BI, Ekholm S. A case of cervical spinal cord glioblastoma diagnosed with MR diffusion tensor and perfusion imaging. J Neuroimaging. 2011; 21:292-6.

5. Jallo GI, Freed D, Epstein F. Intramedullary spinal cord tumors in children. Childs Nerv Syst 2003; 19:641-649

6. Houten JK, Cooper PR. Spinal cord astrocytomas: presentation, management and outcome. J Neurooncol. 2000; 47:219-224.

7. Esau C, Kang X, Peralta E, Hanson E, Marcusson EG, Ravichandran LV, Sun Y, Koo S, Perera RJ, Jain R, Dean NM, Freier SM, Bennett CF, et al. MicroRNA-143 regulates adipocyte differentiation. J Biol Chem. 2004; 279:52361-52365.

8. Takanabe R, Ono K, Abe Y, Takaya T, Horie T, Wada H, Kita T, Satoh N, Shimatsu A, Hasegawa K. Up-regulated expression of microRNA-143 in association with obesity in adipose tissue of mice fed high-fat diet. Biochem Biophys Res Commun. 2008; 376:728-732.

9. He Y, Zhao C, Liu Y, He Z, Zhang Z, Gao Y, Jiang J. MiR-124 Functions as a Tumor Suppressor via Targeting hCLOCK1 in Glioblastoma. Mol Neurobiol. 2016. doi:10.1007/s12035-016-9837-y.

10. Chen Y, Ma C, Zhang W, Chen Z, Ma L. Down regulation of miR-143 is related with tumor size, lymph node metastasis and HPV16 infection in cervical squamous cancer. Diagn Pathol. 2014; 28;9:88.

11. Drebber U, Lay M, Wedemeyer I, Vallböhmer D, Bollschweiler E, Brabender J, Mönig SP, Hölscher AH, Dienes HP, Odenthal M. Altered levels of the oncomicroRNA 21 and the tumor-supressor microRNAs 143 and 145 in advanced rectal cancer indicate successful neoadjuvant chemoradiotherapy. Int J Oncol. 2011; 39:409-15.

12. Li F, Li S, Cheng T. TGF- $\beta 1$ promotes osteosarcoma cell migration and invasion through the miR-143-versican pathway. Cell Physiol Biochem. 2014; 34:2169-79.

13. Liao WL, Liu CY, Chang KP, Chang YS, Chen SJ. MicroRNA deregulation and pathway alterations in nasopharyngeal carcinoma. Br J Cancer. 2009; 100:1002-11.

14. Fisher K, Lin J. MicroRNA in inflammatory bowel disease: Translational research and clinical implication. World J Gastroenterol. 2015; 21:12274-82. 
15. Thomas J, Ohtsuka M, Pichler M, Ling H. MicroRNAs: Clinical Relevance in Colorectal Cancer. Int J Mol Sci. 2015; 16:28063-76.

16. Rajasekaran S, Rajaguru P, Sudhakar Gandhi PS. MicroRNAs as potential targets for progressive pulmonary fibrosis. Front Pharmacol. 2015; 6:254.

17. Wu X, Tan X, Fu SW. May Circulating microRNAs be Gastric Cancer Diagnostic Biomarkers?. J Cancer. 2015; 6:1206-13.

18. Yanokura M, Banno K, Iida M, Irie H, Umene K, Masuda $\mathrm{K}$, Kobayashi Y, Tominaga E, Aoki D. MicroRNAS in endometrial cancer: recent advances and potential clinical applications. EXCLI J. 2015; 14:190-8.

19. Lochhead PA, Gilley R, Cook SJ. ERK5 and its role in tumour development. Biochem Soc Trans. 2012; 40:251-6.

20. Lee J. D, Ulevitch R. J, Han J. Primary structure of BMK1: a new mammalian MAP kinase.Biochem Biophys Res Commun. 1995; 213:715-724.

21. Zhou G, Bao Z. Q, Dixon J. E. Components of a new human protein kinase signal transduction pathway. J Biol Chem. $1995 ; 270: 12665-12669$. 